\title{
The Association between the Extent of Late Gadolinium Enhancement and Diastolic Dysfunction in Hypertrophic Cardiomyopathy
}

\author{
Deniz Alis ${ }^{1} \quad$ Arda Guler $^{2} \quad$ Ozan Asmakutlu ${ }^{3} \quad$ Cagdas Topel $^{3} \quad$ Ahmet A. Sahin ${ }^{2}$
}

${ }^{1}$ Department of Radiology, Acibadem Mehmet Ali Aydinlar University School of Medicine, Istanbul, Turkey

2Department of Cardiology, Istanbul Mehmet Akif Ersoy Thoracic and Cardiovascular Surgery Training and Research Hospital, Halkali/ Istanbul, Turkey

${ }^{3}$ Department of Radiology, Istanbul Mehmet Akif Ersoy Thoracic and Cardiovascular Surgery Training and Research Hospital, Halkali/ Istanbul, Turkey
Address for correspondence Deniz Alis, Department of Radiology, Acibadem Mehmet Ali Aydinlar University School of Medicine, Istanbul, Turkey (e-mail: drdenizalis@gmail.com).

Indian J Radiol Imaging 2021;31:284-290.

\begin{abstract}
Keywords

- atrial volume

- CMR

- diastolic dysfunction

- hypertrophic cardiomyopathy

- LGE

Background Diastolic dysfunction in hypertrophic cardiomyopathy (HCM) patients is a frequent, yet poorly understood phenomenon.

Purpose The purpose of this study is to assess the relationship between the myocardial fibrosis and diastolic dysfunction in patients with HCM.

Materials and Methods We retrospectively investigated the impact of the myocardial fibrosis, as assessed by the extent of late gadolinium enhancement (LGE-\%) on cardiac magnetic resonance imaging (CMRI), on diastolic dysfunction in 110 patients with HCM. The diastolic dysfunction was evaluated by the left atrial (LA) volume index measured on CMRI and lateral septal E/E' ratio calculated on echocardiography.

Results : There was a moderate correlation between the LGE-\% and LA volume $(r=0.59, p<0.0001)$. The logistic regression model of LGE-\%, mitral regurgitation, and total left ventricular mass that investigated the independent predictors of LA volume identified LGE-\% as the only independent parameter associated with the LA volume index $(\beta=0.30, p=0.003)$. No correlation was observed between the LGE-\% and $E / E^{\prime}$ $(r=0.24, p=0.009)$.

Conclusions Myocardial fibrosis in HCM patients is associated with a chronic diastolic burden as represented by increased LA volume. However, the fibrosis does not influence the $E / E^{\prime}$ ratio, which is a well-known parameter of ventricular relaxation, restoring forces, and filling pressure.
\end{abstract}

\section{Introduction}

Hypertrophic cardiomyopathy (HCM) is a genetic disease of the myocardium which occurs as a result of mutations in genes encoding protein components of the cardiac sarcomere. ${ }^{1-3}$

published online July 28, 2021
DOI https://doi.org/

$10.1055 / \mathrm{s}-0041-1734333$ ISSN 0971-3026
The disease is characterized by marked hypertrophy of the myocardium, myofibrillary disarray, and increased amount of myocardial collagen. ${ }^{1-3}$ Most HCM patients, to some extent, had a variable degree of diastolic dysfunction, and diastolic dysfunction is associated with adverse outcomes. ${ }^{4}$
(C) 2021. Indian Radiological Association.

This is an open access article published by Thieme under the terms of the Creative Commons Attribution-NonDerivative-NonCommercial-License, permitting copying and reproduction so long as the original work is given appropriate credit. Contents may not be used for commercial purposes, or adapted, remixed, transformed or built upon. (https://creativecommons.org/licenses/by-nc-nd/4.0/).

Thieme Medical and Scientific Publishers Private Ltd. A-12, Second Floor, Sector -2, NOIDA -201301, India 
Diastolic dysfunction in HCM is thought to occur due to the interplay of complex factors; hence, no single noninvasive modality could readily detect the presence of diastolic dysfunction in HCM..$^{5-9}$ Replacement fibrosis and increased extracellular collagen in HCM hampers the elastic properties of the normal myocardium, impairs relaxation, and increases passive stiffness. ${ }^{5-9}$ Diastolic dysfunction in HCM patients might precede systolic dysfunction, which could eventually result in heart failure..$^{5-9}$ Therefore, prompt and precise diagnosis of diastolic dysfunction in HCM patients is appealing.

Echocardiography is the most commonly used modality for the evaluation of the ventricular and atrial mechanics in HCM patients. Echocardiography has an excellent temporal resolution, yet is limited by poor acoustic windows, inability to perform multiplanar imaging, and low interobserver reproducibility. ${ }^{10}$ Cardiac magnetic resonance imaging (CMRI) is an emerging modality and is being increasingly used in the assessment of HCM. ${ }^{11}$ CMRI had multiplanar imaging capacity with high spatial and contrast resolution. ${ }^{11}$ Furthermore, late gadolinium enhancement (LGE) on CMRI enables to indirectly determine the fibrosis changes that occurred in the myocardium in patients with HCM as validated by radiopathological correlation studies. ${ }^{11,12}$

Herein, the present work aimed to investigate the impact of myocardial fibrosis on diastolic functions in HCM.

\section{Materials and Methods}

The local ethics committee approved this retrospective study conducted between January 2015 and January 2019. The institutional board waived the need for informed consent for the use of deidentified medical and clinical data of the patients. We searched our database to identify patients with HCM diagnoses which were established according to European Society of Cardiology (ESC) Guidelines on diagnosis and management of HCM. ${ }^{3}$ The inclusion criteria were the following: (1) having CMRI with LGE sequence and (2) having echocardiography performed within 3 months before or after the CMRI. The exclusion criteria were the followings: (1) history of myocardial infarct, (2) the presence or history of coronary artery disease, (3) history of any autoimmune or storage disorder, (4) the presence of hypertension, and (5) history of rheumatologic valvular disease.

The diastolic dysfunction was assessed according to the "Recommendations for the Evaluation of Left Ventricular Diastolic Function by Echocardiography" offered by the American Society of Echocardiography and the European Association of Cardiovascular Imaging. ${ }^{13}$ The guideline recommends an in-depth approach when assessing diastolic dysfunction in patients with HCM and suggests several methods for the evaluation of diastolic dysfunction in HCM patients. The present study implemented $\mathrm{E} / \mathrm{E}^{\prime}$ ratio and left atrial (LA) volume index as the representative imaging parameters for diastolic dysfunction. In the present work, the $E / E^{\prime}$ ratio was calculated on echocardiography, while the LA volume index was calculated on CMRI.

\section{Echocardiographic Examination}

A single observer with more than 5 years of echocardiography experience conducted all transthoracic echocardiography examinations with a standard clinical protocol. The observer performed all examinations using an S5-1 transducer with the same ultrasound unit (Philips EPIQ 7 system, Philips Medical Systems, Bothell, Washington, United States). Peak velocities were obtained at early (E) and late diastole (A). Tissue Doppler imaging was employed at the lateral aspect of the mitral annulus on the apical four-chamber view, and $\mathrm{E}^{\prime}$ value was calculated using $\mathrm{cm} / \mathrm{s}$ as a unit. The observer calculated $\mathrm{E} / \mathrm{E}^{\prime}$, which is an accurate parameter in estimating relatively load-independent LV filling pressure. ${ }^{13}$

\section{Cardiac Magnetic Resonance Acquisitions}

All MRI studies were acquired with a $1.5 \mathrm{~T}$ scanner (Aera, Siemens Medical Systems, Enlargen, Germany). All CMR acquisitions were performed using phased-array body coils. All of the sequences were acquired using prospective cardiac gating. Our CMR protocol in the order of first to latest consisted of breath-hold black-axial blood fast spin echo (SE), multiple breath-hold long-axis four-chamber, long axis two-chamber, and 9 to 12 stack of short axes cine images breath-hold using balanced steady-state free precession imaging (SSFP), and LGE sequences in four-chamber, two-chamber, and short-axis views covering entire left ventricle myocardium. LGE sequences obtained approximately 12 minutes (ranging: 10-15 minutes) after the administration of 0.20 to $0.22 \mathrm{mmol} / \mathrm{kg}$ gadopentetate dimeglumine (Magnevist, Schering AG, Berlin, Germany). The parameters for SSFP cine images were the followings: $\mathrm{TR} / \mathrm{TE}=3.8 / 1$ to $3 \mathrm{~ms}$, slice thickness $=5 \mathrm{~mm}$ with $5-\mathrm{mm}$ interslice gap, temporal resolution $=35 \mathrm{~m}$, and parameters for LGE sequences were the followings: TR/TE $=9 / 3 \mathrm{~ms}$, slice thickness $=5 \mathrm{~mm}$, inversion time $=200$ to $300 \mathrm{~ms}$ adjusted according to patient to completely null the normal myocardial signal. Total acquisition time ranged between 40 and 60 minutes.

\section{Cardiac Magnetic Resonance Image Analyses}

The CMRI of the patients were retrieved from our hospital picture archiving and communicating system (PACS, Extremepacs system Ankara, Turkey).

A single radiologist (D.A.) with 4 years of CMRI interpretation experience evaluated all the CMR images. Ejection fraction (EF), end-systolic volume, end-diastolic volume, stroke volume, cardiac index, cardiac output, and total left ventricular mass (TLVM) were calculated and indexed to body surface area (BSA) using modified Simpson's method on short-axis cine images (ARGUS, Siemens, Erlangen, Germany). The maximal left myocardial thickness was assessed using the American Heart Association 16-segment model as 6 regions at the basal level, 6 regions at the midventricular level, and 4 regions at the apical level. ${ }^{14}$ The observer perpendicularly measured the maximal wall thickness on short-axis cine images during end diastole. The LA volume was calculated 
using the biplanar method at the end systole (volume $=[0.85$ $\times$ four-chamber area $\times$ two-chamber area] / length of the perpendicular axis) and then proportioned to the BSA.

Afterward, the observer assessed the presence and the extent of myocardial fibrosis on LGE images. The left ventricular myocardium was evaluated on short-axis images. The observers manually delineated the endocardial and epicardial borders. The area with LGE was quantitatively measured using a visually determined threshold to cover areas with high and intermediate signal intensities. The extent of myocardial
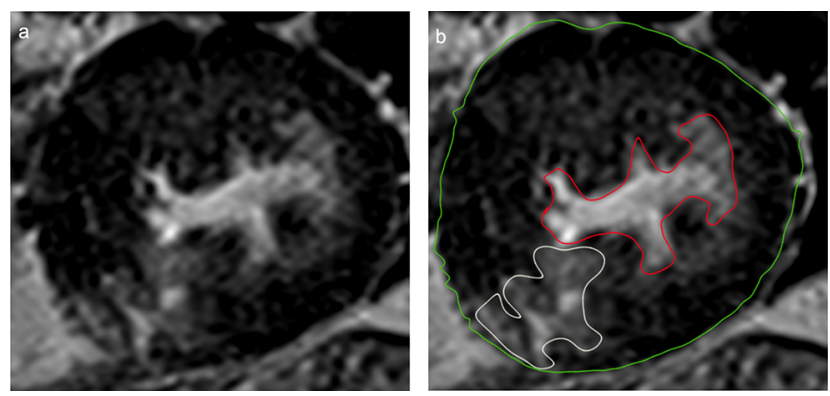

Fig. 1 (A and B) An asymmetric hypertrophic cardiomyopathy patient with focal late gadolinium enhancement in the inferior septum at the mid-ventricular level $(\mathbf{A})$. The manual delineations of epicardial (green), endocardial (red) borders and the area with intermediate to high-signal intensity representing fibrosis are shown (white) (B). The extent of myocardial fibrosis was calculated by proportioning the LGE (+) areas to whole myocardial mass by on the short-axis images and was noted as LGE-\% for each patient. LGE, late gadolinium enhancement. fibrosis was calculated by proportioning the LGE (+) areas to TLVM by the short-axis images. The observer recorded the extent of LGE as LGE-\% for each patient. - Fig. 1 depicts the quantification of LGE in a patient with HCM.

\section{Statistical Analyses}

Statistical analyses were performed using the SPSS software version 21. The variables were investigated using ShapiroWilk test to determine whether or not they were normally distributed. Descriptive analyses were presented using the means and the standard deviations for normally distributed variables and the median and the interquartile ranges for nonnormally distributed variables. The impact of the presence of mitral regurgitation, left ventricular maximal wall thickness (LVMWT), TLVM, and the LGE-\% on diastolic dysfunction (LA volume and E/E' ratio) was first assessed by the Pearson's correlation tests for normally distributed continuous variables or the Spearman's correlation test for ordinal or not normally distributed continuous variables. Pearson's product-moment correlation coefficient and Spearman's rank correlation coefficient, denoted by $r$, were interpreted as the followings: the $r$ values of 0.00 to 0.29 as a negligible correlation, 0.30 to 0.49 as a weak correlation, 0.50 to 0.69 as a moderate correlation, 0.70 to 0.90 as a good correlation, and 0.90 to 1 as an excellent correlation. ${ }^{15}$

The variables with a $p$-value of less than 0.05 in univariate analyses were further entered into logistic regression analyses to assess independent predictors of diastolic dysfunction. Hosmer-Lemeshow goodness of fits statistics was used to
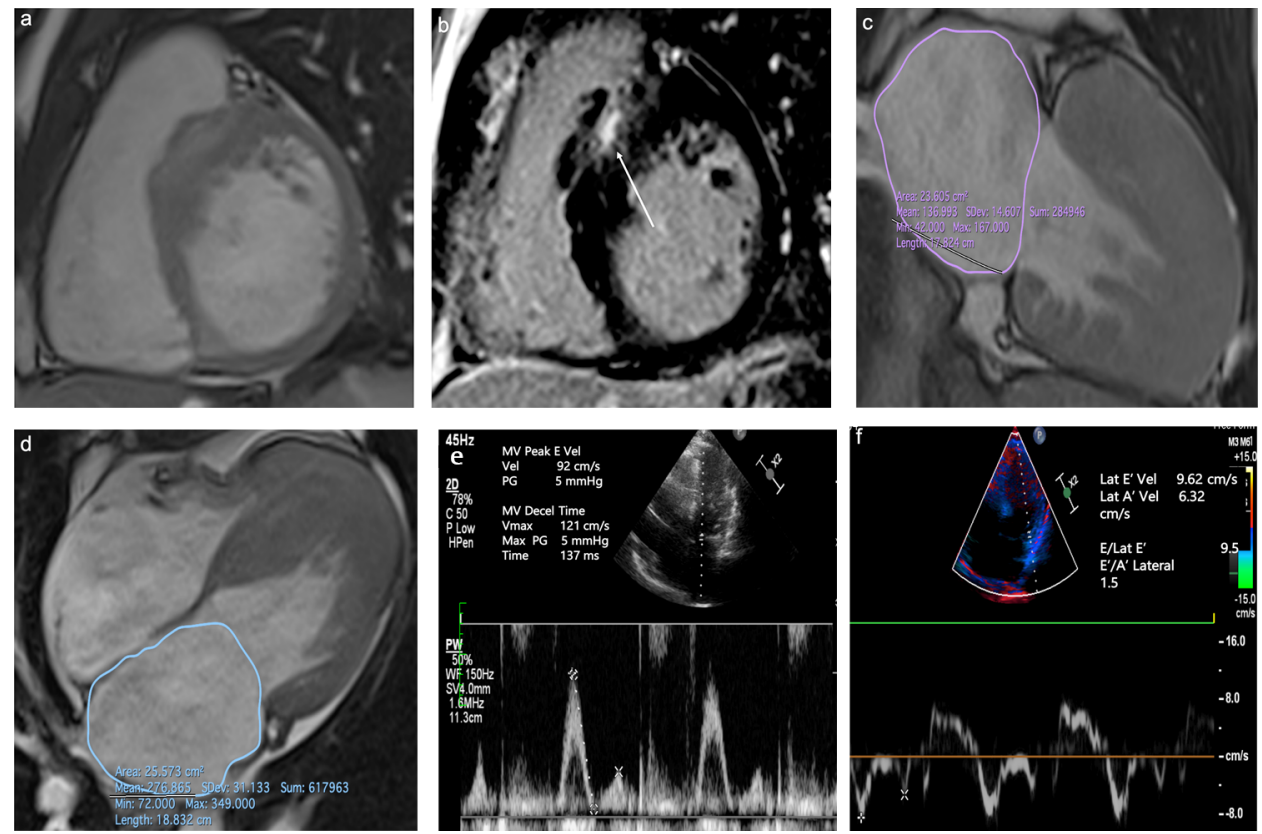

Fig. 2 (A-F) A 40-year-old female patient with hypertrophic cardiomyopathy. Short-axis (A) cardiac magnetic resonance image at end diastole shows marker thickening of the interventricular septum (arrow). The corresponding short-axis late gadolinium image (B) shows patchy contrast enhancement in the thickened wall (arrow). Two-chamber (C) and four-chamber long-axis (D) cardiac magnetic resonance images at end systole reveal prominent left atrium dilatation. The left atrial volume is calculated as $46 \mathrm{~mL} / \mathrm{m}^{2}$ by the biplanar method. The peak E-wave velocity $(\mathrm{cm} / \mathrm{s})$ is calculated as $92 \mathrm{~cm} / \mathrm{s}$ on echocardiography (E). The tissue Doppler imaging depicts lateral $\mathrm{E}^{\prime}$-wave velocity of $9.62 \mathrm{~cm} / \mathrm{s}$. The $\mathrm{E} / \mathrm{E}^{\prime}$ ratio of the patient is calculated as 9.56, which is within normal limits despite the presence of late gadolinium enhancement and left atrial dilatation (F) The tissue Doppler imaging depicts lateral $E^{\prime}$-wave velocity of $9.62 \mathrm{~cm} / \mathrm{s}$. The $E / E^{\prime}$ ratio of the patient is calculated as 9.56 , which is within normal limits despite the presence of late gadolinium enhancement and left atrial dilatation. 
Table 1 Clinical, CMR, and echocardiographic findings of the patients

\begin{tabular}{|c|c|}
\hline Variables & Findings \\
\hline Age (y) & $49.92 \pm 13.26$ \\
\hline \multicolumn{2}{|l|}{ Gender } \\
\hline Male & $71(64.5 \%)$ \\
\hline Female & 39 (35.5\%) \\
\hline \multicolumn{2}{|l|}{ NYHA class } \\
\hline I & 45 (40.9\%) \\
\hline II & $44(40 \%)$ \\
\hline III & $17(15.5 \%)$ \\
\hline IV & $4(3.6 \%)$ \\
\hline \multicolumn{2}{|l|}{ HCM subtype } \\
\hline Asymmetrical septal & $71(64.5 \%)$ \\
\hline Symmetrical septal & $9(7.3 \%)$ \\
\hline Diffuse concentric & $20(18.2 \%)$ \\
\hline Asymmetrical concentric & $7(6.3 \%)$ \\
\hline Midventricular obstructive & $3(2.7 \%)$ \\
\hline End systolic volume $\left(\mathrm{mL} / \mathrm{m}^{2}\right)$ & $17.67 \pm 10.28$ \\
\hline End diastolic volume $\left(\mathrm{mL} / \mathrm{m}^{2}\right)$ & $65.57 \pm 16.69$ \\
\hline Stroke volume $\left(\mathrm{mL} / \mathrm{m}^{2}\right)$ & $50.15 \pm 13.03$ \\
\hline Cardiac index $\left(\mathrm{L} / \mathrm{min} / \mathrm{m}^{2}\right)$ & $3.39 \pm 0.88$ \\
\hline Cardiac output (L/min) & $6.26 \pm 1.73$ \\
\hline Ejection fraction (\%) & $73.86 \pm 10.04$ \\
\hline $\begin{array}{l}\text { Maximal left ventricular wall } \\
\text { thickness }(\mathrm{mm})^{\mathrm{a}}\end{array}$ & $18.35(6.9)$ \\
\hline Left ventricular diameter $(\mathrm{cm})$ & $4.93 \pm 4.35$ \\
\hline Myocardial mass $\left(\mathrm{g} / \mathrm{m}^{2}\right)^{\mathrm{a}}$ & $116.5(56.7)$ \\
\hline$E / E^{b}$ & $10.91 \pm 3.71$ \\
\hline$E / A^{b}$ & $1.23 \pm 0.40$ \\
\hline E wave $(s)^{b}$ & $76.78 \pm 22.95$ \\
\hline A wave $(s)^{b}$ & $71.81 \pm 25.47$ \\
\hline \multicolumn{2}{|l|}{ Mitral insufficiency ${ }^{b}$} \\
\hline Yes & $37(32.7 \%)$ \\
\hline No & $73(66.4 \%)$ \\
\hline \multicolumn{2}{|c|}{ Presence of late gadolinium enhancement } \\
\hline Yes & $72(65.6 \%)$ \\
\hline No & $38(34.4 \%)$ \\
\hline $\begin{array}{l}\text { The extent of late gadolinium } \\
\text { enhancement (\%) }\end{array}$ & $9.32 \pm 3.38$ \\
\hline
\end{tabular}

Abbreviations: CMR, cardiac magnetic resonance; HCM, hypertrophic cardiomyopathy; NYHA, New York Heart Association.

Note: All variables are expressed as mean \pm standard deviations unless otherwise specified.

aExpressed as median (interquartile range).

bEchocardiography-derived parameters. assess model fit. A $p$-value of $<0.05$ was used to infer a statistical significance.

\section{Results}

A total of 110 patients with HCM, 39 females (35.5\%), and 71 males $(64.5 \%)$ with the mean age of $49.92 \pm 13.26$ years, were enrolled in the study cohort. - Table 1 shows the patients' demographics, echocardiography, and CMRI findings in detail. In the study cohort, 72 out of 110 patients (65.6\%) demonstrated LGE, and the LGE-\% was calculated as $9.32 \pm 3.38$. The median LA volume index was 60.23 (33.88). The Spearman's rank correlation showed a moderate positive correlation between LGE-\% and LA volume ( $r=$ $0.59, p<0.0001)$. Additionally, the presence of LGE and LA was also positively correlated as assessed by the Spearman's test $(0.52, p<0.0001)$. There were significant correlations between TLVM, mitral regurgitation, and LA volume ( $p<$ 0.05; - Table 2).

The mean $\mathrm{E} / \mathrm{E}^{\prime}$ ratio of the patients was $10.91 \pm 3.71$. No correlation was observed between LVMWT and LA volume (-Table 2). Pearson's test revealed a negligible correlation between the LGE-\% and E/E' ( $r=0.24, p=0.009)$. However, no correlation was observed between LVMWT, mitral regurgitation, the presence of LGE, and E/E' ratio ( - Table 2). The multivariate analysis of the LGE-\%, the presence of LGE, TLVM, mitral regurgitation, and LA volume in predicting $E / E^{\prime}$ ratio that revealed only LA volume independently associated with $\mathrm{E} / \mathrm{E}^{\prime}([3=0.32, p=0.004]$; - Table 3). The multivariate analysis model of the LGE-\%, the presence of LGE, TLVM, mitral regurgitation, and $\mathrm{E} / \mathrm{E}^{\prime}$ ratio in predicting $\mathrm{LA}$ volume revealed that $\mathrm{LGE}-\%$, the presence of $\mathrm{LGE}$, and $\mathrm{E} / \mathrm{E}^{\prime}$ ratio were independently associated with LA volume ([3=0.30, $p=0.003]$, [3 $=0.25, p=0.018]$, and $[p=0.24, p=0.004]$, respectively). -Fig. 2 shows CMRI and echocardiographic images of an HCM patient who had LGE and increased LA volume index, yet normal E/E' ratio.

A weak positive correlation was observed between the LGE-\% and TLVM ( $r=0.36, p<0.0001)$, and a moderate positive correlation was identified between the presence of LGE and TLVM $(r=0.45, p<0.0001)$. No correlation existed between left ventricular global functions and the extent of LGE as assessed by EF $(r=-0.21, p=0.037)$.

\section{Discussion}

The present study identified a moderate positive correlation between LGE-\% and LA volume, yet no correlation between the LGE-\% and E/E' ratio. Notably, only a weak positive correlation was observed between two different methods in assessing diastolic dysfunction, LA volume index and $E / E^{\prime}$ ratio.

There is a solid body of literature on the diagnostic value of LGE in estimating diastolic dysfunction. ${ }^{16-19}$ However, many of these studies did not assess the LA volume index which is a recognized marker of chronic diastolic burden. ${ }^{20} \mathrm{~A}$ report by the American College of Cardiology Foundation/American Heart Association Task Force for the diagnosis and treatment 
Table 2 Univariate correlations between CMR parameters, LA volume, and E/E'

\begin{tabular}{|l|l|l|l|l|}
\hline \multirow{2}{*}{ Parameters } & \multicolumn{2}{|c|}{ LA volume } & \multicolumn{2}{c|}{ E/E' } \\
\cline { 2 - 5 } & $r$ & $p$ & $r$ & $p$ \\
\hline LGE-\% & 0.59 & $<0.0001$ & 0.24 & 0.009 \\
\hline Presence of LGE & 0.52 & $<0.0001$ & 0.16 & 0.081 \\
\hline Mitral regurgitation & 0.26 & 0.006 & 0.16 & 0.09 \\
\hline E/E' & 0.37 & $<0.0001$ & NA & NA \\
\hline LA volume $\left(\mathrm{mL} / \mathrm{m}^{2}\right)$ & NA & NA & 0.37 & $<0.0001$ \\
\hline TLVM $\left(\mathrm{g} / \mathrm{m}^{2}\right)$ & 0.29 & 0.002 & 0.25 & 0.008 \\
\hline LVMWT $(\mathrm{mm})$ & 0.18 & 0.057 & 0.62 & 0.52 \\
\hline
\end{tabular}

Abbreviations: CMR, cardiac magnetic resonance; LA, left atrium; LGE, late gadolinium enhancement; LVMWT, left ventricular maximal wall thickness; NA, not applicable; TLVM, total left ventricular mass.

Table 3 Multivariate analyses assessing independent predictors of left atrial volume and E/E' ratio

\begin{tabular}{|l|l|l|l|l|}
\hline \multirow{2}{*}{} & \multicolumn{2}{|c|}{ LA volume } & \multicolumn{2}{c|}{ E/E' } \\
\cline { 2 - 5 } & OR & $P$ & OR & 0.10 \\
\hline LGE-\% & 0.30 & 0.003 & 0.19 & 0.17 \\
\hline Presence of LGE & 0.25 & 0.018 & 0.16 & 0.27 \\
\hline Mitral regurgitation & 0.09 & 0.24 & 0.10 & 0.088 \\
\hline TLVM $\left(\mathrm{g} / \mathrm{m}^{2}\right)$ & 0.32 & 0.77 & 0.16 & 0.004 \\
\hline LA volume $\left(\mathrm{mL} / \mathrm{m}^{2}\right)$ & NA & NA & 0.32 & NA \\
\hline E/E' & 0.24 & 0.004 & NA & \\
\hline
\end{tabular}

Abbreviations: CMR, cardiac magnetic resonance; LA, left atrium; LGE, late gadolinium enhancement; NA, not applicable; OR, odds ratio; TLVM, total left ventricular mass.

of HCM highlights that LA volume can predict exercise capacity in HCM patients and can also reflect chronic diastolic burden. ${ }^{21}$ Furthermore, this report suggests that increased LA volume is associated with less favorable clinical outcome. ${ }^{21}$ The findings of the present study pointed out to a moderate positive correlation between the LGE-\% and LA volume in HCM patients in line with several previous reports. ${ }^{22}$

The research regarding the association between LGE-\% and $\mathrm{E} / \mathrm{E}^{\prime}$ ratio is inconclusive. Zhu et $\mathrm{a}^{23}$ evaluated the correlation between $\mathrm{LGE}-\%$ and $\mathrm{E} / \mathrm{E}^{\prime}$ ratio in 61 patients with $\mathrm{HCM}$. The authors identified a moderate positive correlation between the extent of LGE and E/E' ratio in univariate analysis, yet this relationship did not reach significance in their multivariate model. ${ }^{23}$ However, Zhu et al ${ }^{23}$ identified a positive correlation between the LGE-\% in the ventricular insertion points and $\mathrm{E} / \mathrm{E}^{\prime}$ ratio. Choi et $\mathrm{al}^{22}$ failed to demonstrate any association between the LGE-\% and E/E' ratio. In corroboration with the work by Choi et al, the present study identified no correlation between LGE-\% and E/E' ratio. Most intriguingly, there was only a weak positive correlation between LA volume and $E / E^{\prime}$ ratio in the present work.

A possible explanation for these results may be related to the comprehensive nature of the diastolic function which could be influenced by many factors, including ventricular load, ischemic process, heart rate, and systolic emptying. ${ }^{13,17}$ LA volume index is primarily a surrogate marker of long-term elevation of LA pressure and volume. E/E' ratio mainly reflects the LV relaxation, restoring forces, and filling pressures. LGE in HCM mostly occurs due to macroscopic replacement fibrosis and scar tissue rather than diffuse interstitial fibrosis. ${ }^{24}$ Hence, we suggest that LGE-\% might not be a proper marker for $\mathrm{LV}$ relaxation as assessed by the $\mathrm{E} / \mathrm{E}^{\prime}$ ratio, whereas it might be related to the chronic diastolic burden in HCM patients. The findings of the study by Ellims et al ${ }^{19}$ supported this argument. The authors employed LGE and Tl mapping to patients with HCM for assessing diastolic dysfunction, and only the marker of diffuse interstitial fibrosis, prolonged $\mathrm{Tl}$ time, had influence on $E / E^{\prime}$ ratio, yet there was no correlation between the macroscopic replacement fibrosis and scar tissue, as assessed by LGE and E/E' ratio. ${ }^{19}$ Nevertheless, we suggest that much research needs to be performed on the association between the LGE-\% and E/E' ratio with the utmost integrity before establishing a solid conclusion regarding this relationship.

The present work shows that TLVM did not correlate with $\mathrm{E} / \mathrm{E}^{\prime}$ ratio and LA volume, and there were only weak positive correlations between LVMWT with $\mathrm{E} / \mathrm{E}^{\prime}$ ratio and LA volume. Zhu et $\mathrm{al}^{23}$ also found no correlation between diastolic dysfunction with LVMWT and TLVM. Ellims et $\mathrm{al}^{19}$ found that TLVM and LVMWT had weak negative correlations with E/E' ratio which failed to reach statistical significance in the multivariate model. Hence, one might suggest that TLVM and LVMWT do not seem to be good indicators of diastolic dysfunction in HCM patients.

\section{Limitations}

We had several drawbacks in the present work. First, the present study did not assess recognized CMR parameters 
for diastolic dysfunction assessment, such as left ventricular volume curves or employ mitral and pulmonary vein phase velocity imaging. Nonetheless, previous reports have demonstrated the superiority of echocardiograph-based velocity $\mathrm{E} / \mathrm{E}^{\prime}$ measurements over CMR-based flow parameters given to the native higher temporal resolution of echocardiography. ${ }^{18}$ Hence, we suggest that this issue does not hamper the reliability of our results. Second, the reference method in estimating diastolic function is direct catheterization, yet we did not employ any invasive measurement techniques in the present work. However, previous works demonstrated that $\mathrm{E} / \mathrm{E}^{\prime}$ is well correlated with the catheter-based direct pressure measurements. ${ }^{25}$ Third, given to the retrospective nature of the work, we could not assess whether LGE-\% was associated with long-term clinical outcomes of the patients or could not assess the relationship of LA volume and $\mathrm{E} / \mathrm{E}^{\prime}$ ratio with the prognosis of patients.

\section{Conclusion}

The present study shows that LGE-\% is associated with the chronic diastolic burden in HCM patients. However, no correlation was observed between $\mathrm{LGE}-\%$ and $\mathrm{E} / \mathrm{E}^{\prime}$ ratio, which is a well-known parameter of LV relaxation, restoring forces, and filling pressure.

\section{Ethical Statement and Consent to Participate}

All procedures performed in studies involving human participants were in accordance with the ethical standards of the institutional and/or national research committee and with the 1964 Helsinki declaration and its later amendments or comparable ethical standards.

\section{Financial Support and Sponsorship}

None.

\section{Conflicts of Interest}

There are no conflicts of interest.

\section{References}

1 Maron BJ. Hypertrophic cardiomyopathy: a systematic review. JAMA 2002;287(10):1308-1320

2 Wigle ED, Rakowski H, Kimball BP, Williams WG. Hypertrophic cardiomyopathy. Clinical spectrum and treatment. Circulation 1995;92(7):1680-1692

3 Elliott PM, Anastasakis A, Borger MA, et al. Authors/Task Force members. 2014 ESC Guidelines on diagnosis and management of hypertrophic cardiomyopathy: the Task Force for the Diagnosis and Management of Hypertrophic Cardiomyopathy of the European Society of Cardiology (ESC. Eur Heart J 2014;35(39):2733-2779

4 Fifer MA, Vlahakes GJ. Management of symptoms in hypertrophic cardiomyopathy. Circulation 2008;117(3):429-439

5 Lester SJ, Tajik AJ, Nishimura RA, Oh JK, Khandheria BK, Seward JB. Unlocking the mysteries of diastolic function: deciphering the Rosetta Stone 10 years later. J Am Coll Cardiol 2008;51(7):679-689

6 Kass DA, Bronzwaer JG, Paulus WJ. What mechanisms underlie diastolic dysfunction in heart failure? Circ Res 2004;94(12):1533-1542
7 Geske JB, Sorajja P, Nishimura RA, Ommen SR. Evaluation of left ventricular filling pressures by Doppler echocardiography in patients with hypertrophic cardiomyopathy: correlation with direct left atrial pressure measurement at cardiac catheterization. Circulation 2007;116(23):2702-2708

8 Rakowski H, Carasso S. Quantifying diastolic function in hypertrophic cardiomyopathy: the ongoing search for the holy grail. Circulation 2007;116(23):2662-2665

9 Sipola P, Lauerma K, Jääskeläinen P, et al. Cine MR imaging of myocardial contractile impairment in patients with hypertrophic cardiomyopathy attributable to Asp175Asn mutation in the alpha-tropomyosin gene. Radiology 2005;236(3):815-824

10 Pons-Lladó G, Carreras F, Borrás X, Palmer J, Llauger J, Bayés de Luna A. Comparison of morphologic assessment of hypertrophic cardiomyopathy by magnetic resonance versus echocardiographic imaging. Am J Cardiol 1997;79(12):1651-1656

11 Pennell DJ. Cardiovascular magnetic resonance. Circulation 2010;121(5):692-705

12 Reichek N, Gupta D. Hypertrophic cardiomyopathy: cardiac magnetic resonance imaging changes the paradigm. J Am Coll Cardiol 2008;52(7):567-568

13 Nagueh SF, Smiseth OA, Appleton CP, et al. Houston, Texas; Oslo, Norway; Phoenix, Arizona; Nashville, Tennessee; Hamilton, Ontario, Canada; Uppsala, Sweden; Ghent and Liège, Belgium; Cleveland, Ohio; Novara, Italy; Rochester, Minnesota; Bucharest, Romania; and St. Louis, Missouri. Recommendations for the evaluation of left ventricular diastolic function by echocardiography: an update from the American Society of Echocardiography and the European Association of Cardiovascular Imaging. Eur Heart J Cardiovasc Imaging 2016;17(12):1321-1360

14 Cerqueira MD, Weissman NJ, Dilsizian V, et al. American Heart Association Writing Group on Myocardial Segmentation and Registration for Cardiac Imaging. Standardized myocardial segmentation and nomenclature for tomographic imaging of the heart. A statement for healthcare professionals from the Cardiac Imaging Committee of the Council on Clinical Cardiology of the American Heart Association. Circulation 2002;105(4):539-542

15 Hinkle DE, Wiersma W, Jurs SG, Applied Statistics for the Behavioral Sciences 5th ed. Boston, Massachusetts: Houghton Mifflin; 2003

16 Motoyasu M, Kurita T, Onishi K, et al. Correlation between late gadolinium enhancement and diastolic function in hypertrophic cardiomyopathy assessed by magnetic resonance imaging. Circ J 2008;72(3):378-383

17 Nucifora G, Muser D, Gianfagna P, Morocutti G, Proclemer A. Systolic and diastolic myocardial mechanics in hypertrophic cardiomyopathy and their link to the extent of hypertrophy, replacement fibrosis and interstitial fibrosis. Int J Cardiovasc Imaging 2015;31(8):1603-1610

18 Rathi VK, Biederman RWW. Expanding role of cardiovascular magnetic resonance in left and right ventricular diastolic function. Heart Fail Clin 2009;5(3):421-435, vii

19 Ellims AH, Iles LM, Ling LH, Hare JL, Kaye DM, Taylor AJ. Diffuse myocardial fibrosis in hypertrophic cardiomyopathy can be identified by cardiovascular magnetic resonance, and is associated with left ventricular diastolic dysfunction. J Cardiovasc Magn Reson 2012;14:76

20 Yang H, Woo A, Monakier D, et al. Enlarged left atrial volume in hypertrophic cardiomyopathy: a marker for disease severity. J Am Soc Echocardiogr 2005;18(10):1074-1082

21 Gersh BJ, Maron BJ, Bonow RO, et al; American College of Cardiology Foundation/American Heart Association Task Force on Practice Guidelines. 2011 ACCF/AHA guideline for the diagnosis and treatment of hypertrophic cardiomyopathy: a report of the American College of Cardiology Foundation/American 
Heart Association Task Force on Practice Guidelines. Developed in collaboration with the American Association for Thoracic Surgery, American Society of Echocardiography, American Society of Nuclear Cardiology, Heart Failure Society of America, Heart Rhythm Society, Society for Cardiovascular Angiography and Interventions, and Society of Thoracic Surgeons. J Am Coll Cardiol 2011;58(25):e212-e260

22 Choi DS, Ha JW, Choi B, et al. Extent of late gadolinium enhancement in cardiovascular magnetic resonance and its relation with left ventricular diastolic function in patients with hypertrophic cardiomyopathy. Circ J 2008;72(9):1449-1453
23 Zhu Y, Park EA, Lee W, et al. Extent of late gadolinium enhancement at right ventricular insertion points in patients with hypertrophic cardiomyopathy: relation with diastolic dysfunction. Eur Radiol 2015;25(4):1190-1200

24 Hansen MW, Merchant N. MRI of hypertrophic cardiomyopathy: part I, MRI appearances. AJR Am J Roentgenol 2007;189(6):1335-1343

25 Kasner M, Westermann D, Steendijk P, et al. Utility of Doppler echocardiography and tissue Doppler imaging in the estimation of diastolic function in heart failure with normal ejection fraction: a comparative Doppler-conductance catheterization study. Circulation 2007;116(6):637-647 